\title{
Change in patient concerns following total knee arthroplasty described with the International Classification of Functioning, Disability and Health: a repeated measures design
} Ravi Rastogi*1, Bert M Chesworth ${ }^{2}$ and Aileen M Davis ${ }^{3}$

Address: ${ }^{1}$ Physiotherapist, London Health Sciences Centre, London, Ontario, Canada, ${ }^{2}$ Assistant Professor, School of Physical Therapy, Faculty of Health Sciences and Department of Epidemiology and Biostatistics, Schulich School of Medicine and Dentistry, The University of Western Ontario, London, Ontario, Canada and ${ }^{3}$ Senior Scientist, Health Care and Outcomes Research Division and Arthritis Community Research Evaluation Unit, Toronto Western Research Institute; Associate Professor, University of Toronto, Toronto, Ontario, Canada

Email: Ravi Rastogi* - ravi.rastogi@lhsc.on.ca; Bert M Chesworth - bcheswor@uwo.ca; Aileen M Davis - adavis@uhnresearch.ca

* Corresponding author

Published: II December 2008

Health and Quality of Life Outcomes 2008, 6:1/2 doi:10.1 I86/1477-7525-6-1/2

This article is available from: http://www.hqlo.com/content/6/1/1/2

(c) 2008 Rastogi et al; licensee BioMed Central Ltd.

This is an Open Access article distributed under the terms of the Creative Commons Attribution License (http://creativecommons.org/licenses/by/2.0), which permits unrestricted use, distribution, and reproduction in any medium, provided the original work is properly cited.

\begin{abstract}
Background: There is no published evidence of how patient concerns change during the first six weeks following total knee arthroplasty (TKA). An understanding of the recovery process from the patient's perspective will inform clinicians on how to best educate patients about their postoperative concerns. Our objectives were to (I) quantify the level of importance for each of 32 previously identified concerns pre-operatively, and across the first six weeks following primary TKA and, (2) convey this change in importance post-operatively using the components of the International Classification of Functioning, Disability and Health (ICF).

Methods: The objectives were achieved using a repeated measures design. Convenience sampling was used to recruit 54 consecutive patients undergoing primary TKA at a hospital in Ontario, Canada. Pre-operatively and at two, four and six weeks post-operatively subjects rated the level of importance for each of the 32 previously identified patient concerns

Results: The importance rating of patient concerns in all four ICF components changed from before surgery to two weeks after surgery. Patient concerns in the Participation component became increasingly important after the first two weeks following surgery. Post-operatively from week two to week four, changes in importance ratings were also found in the Body Function and Activity components, but not in the Environmental Factors component.

Conclusion: Changes in patient concerns mirror their early recovery from TKA surgery. Consistent with this, Participation restrictions become increasingly important to patients after discharge from acute care suggesting that clinicians should think of managing patient expectations for return to societal roles early in post-operative rehabilitation.
\end{abstract}

\section{Background}

Osteoarthritis (OA) of the knee is a common cause of pain and disability in the elderly population [1]. Depending on the severity of the disability, total knee arthroplasty (TKA) has been shown to be an effective procedure in reducing pain, improving function and quality of life in individuals 
suffering from $\mathrm{OA}$ of the knee [2,3]. According to the Canadian Joint Replacement Registry [4], the number of TKA procedures in Canada increased by more than $100 \%$ from $1994 / 95$ to $2004 / 05$ with two-thirds of all knee replacements in the 65-84 year age group. As the baby boomer population approaches this age group, it is expected that this trend will continue.

When evaluating the success of any treatment, opinions of patients are of great significance [5]. This is especially important for elective procedures, such as TKA, which are normally performed to improve the individual's quality of life [6]. Different researchers have demonstrated that patient expectations play an important role in determining the outcome following TKA [7]. To the extent that patient expectations and concerns may be related, it is essential that health care providers appropriately address concerns that are important to patients to maximize outcomes following TKA.

Even though patients are routinely referred for physical therapy in the acute post-operative phase (0-6 weeks) after TKA, there is no published evidence of how patient concerns change during this early period of recovery. An understanding of the recovery process from the patient's perspective will inform the clinician's approach to patient education during rehabilitation.

Many of the outcome measures used to quantify change following primary TKA are based on patients' perceptions of their status, but the choice of outcomes is rarely grounded in a conceptual framework [8,9]. The International Classification of Functioning, Disability and Health (ICF) released by the World Health Organization [10]) provides a unified framework for evaluating health and health-related states of populations and also provides a common language for communication among health care professionals $[11,12]$. The ICF model includes two parts, with each part containing separate components. The first part covers functioning and disability and includes the components of Body Structure and Function, Activities, and Participation. The second part covers contextual factors and includes the components of Environmental Factors and Personal Factors. The World Health Organization [10] has indicated that it is difficult to differentiate between Activities and Participation as defined by the ICF. Others believe that it is necessary to differentiate between these components if the ICF is to be widely accepted to describe functioning in rehabilitation $[13,14]$.

In an earlier study, to identify concerns that patients had about their recovery from surgery, we performed individual face-to-face cross-sectional interviews with 30 patients during the first six weeks following primary TKA for OA. All patient concerns were identified by asking patients to answer one specific question, "What is important to you right now with respect to your recovery from knee replacement surgery?". During the interview it was emphasized that patients should only identify what was important during that particular week of the interview. Patients were allowed to report as many concerns as they wished, all of which were recorded verbatim in writing. The primary investigator (RR) conducted all interviews. We grouped patient comments on the basis of common themes, identifying 32 concerns about surgical recovery that we then linked to the components of the ICF [15]. Seven of these concerns were linked to the Body Function component of the ICF while 15, 4 and 4 concerns were linked to the Activity, Participation and Environmental Factors components, respectively. Receiving appropriate information regarding what to expect with rehabilitation following surgery and being independent were the only two concerns that could not be linked to the ICF. The primary objectives of this study were to (1) quantify the level of importance for each of these concerns pre-operatively and across the first six weeks following primary TKA and, (2) convey this change in importance post-operatively using the components of the ICF.

\section{Methods}

Participants were English-speaking ambulatory patients with knee OA who were waiting for a primary TKA. Sample size calculations were based on a concurrent study of responsiveness of the WOMAC [unpublished]. Convenience sampling was used to recruit consecutive patients from the waiting lists of orthopaedic surgeons working in a large tertiary care hospital. Each subject participated in four evaluation sessions: pre-operatively and at two, four and six weeks after surgery. During each evaluation session subjects rated the level of importance for each of the 32 concerns identified in our earlier study [15] and were also given the opportunity to provide additional concerns. Importance was measured on a seven-point scale (1 $=$ not important at all, $2=$ important to a very small extent, $3=$ important to a small extent, $4=$ important to a moderate extent, $5=$ important to a fairly great extent, $6=$ important to a great extent, $7=$ important to a very great extent). At each evaluation session, subjects also completed the Numeric Pain Rating Scale (NPRS) [16] and the Knee Injury and Osteoarthritis Outcome Score (KOOS) [17] to measure knee-related pain intensity and patient perceived health status, respectively. The Western Ontario McMaster Universities Osteoarthritis Index (WOMAC) is embedded within the KOOS. Therefore, scores for the subscales of the WOMAC were extracted from the KOOS. Both the KOOS and the WOMAC have good psychometric properties and are commonly used to assess patient perceived health status among TKA recipients $[17,18]$. Demographic and clinical characteristics including comorbidities using the Self-Administered Comorbidity 


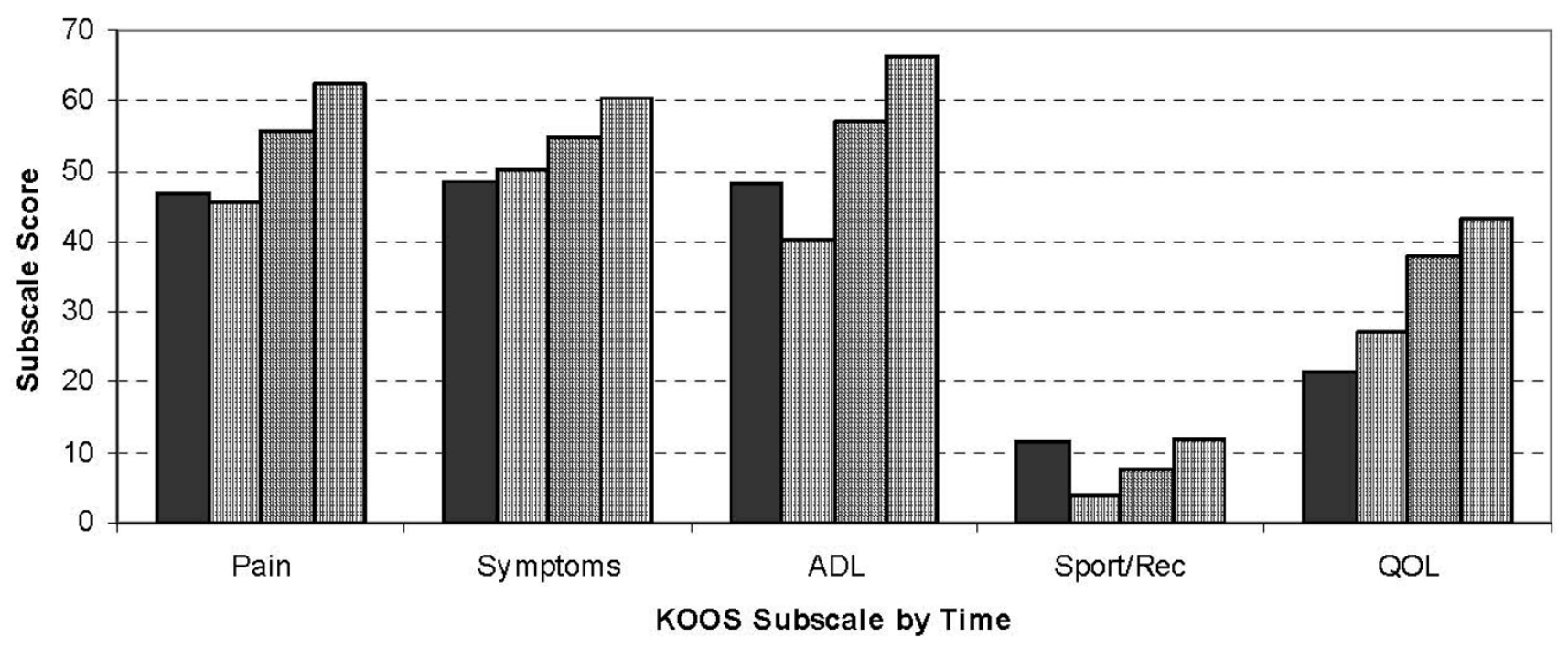

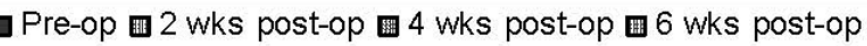

Figure I

Average KOOS* score grouped by subscale: pre-operatively (pre-op) and two, four and six weeks after (postop) primary total knee arthroplasty $(\mathbf{n}=\mathbf{5 4})$. * KOOS = Knee Injury and Osteoarthritis Outcome score: subscales are Pain, other Symptoms, function in daily living (ADL), function in sport and recreation (Sport/Rec) and knee-related Quality of life (QOL). Subscale scores can vary from 0-100 with 100 indicating the best state.

for the two concerns that were not covered by the ICF (receiving appropriate information regarding what to expect with rehabilitation following your surgery and being independent) had high levels of importance at all four time points (i.e. either 6 or 7 on the 7 -point scale).

The Friedman's ANOVA results are shown in Figure 2. The mean rank of the importance rating changed over time ( $\mathrm{p}$ $<0.0001$ ) for all four ICF components (Body Function: $\chi 23 \mathrm{df}=34.29$; Activity: $\chi 23 \mathrm{df}=20.61$; Participation: $\chi 23 \mathrm{df}=90.91$; Environmental Factors: $\chi 23 \mathrm{df}=14.37)$. Post-hoc testing revealed a significant change in importance between the pre-operative evaluation and post- operative week two for all four ICF components ( $\mathrm{p}<$ 0.0001 ). Participation was the only ICF component that demonstrated a significant change in importance between all four-time points $(\mathrm{p}<0.0001)$. The importance of the Body Function and Activity components changed significantly from before surgery through to post-operative week four $(\mathrm{p}<0.001)$.

\section{Discussion}

This study was conducted to gain a quantitative understanding of how the importance of patient concerns change during the first six weeks following primary TKA. It showed that the importance of some concerns change

Table 2: Importance levels for patient concerns linked to the ICF* Body Function component: pre-operatively (pre-op) and two, four and six weeks after knee arthroplasty $(n=54)$

\begin{tabular}{|c|c|c|c|c|}
\hline Patient Concern & Pre-op & Week 2 & Week 4 & Week 6 \\
\hline Decreasing pain in your surgical knee & $6(5-7) \dagger$ & $7(6-7)$ & $6(5-7)$ & $6(4-6)$ \\
\hline Reducing the swelling in your surgical leg & $3(1-6)$ & $6(5-7)$ & $6(5-7)$ & $5(3-6)$ \\
\hline Avoiding infection in your surgical knee & $2(1-7)$ & $7(6-7)$ & $5(2-7)$ & $3(1-6)$ \\
\hline Sleeping better at night & $5(4-7)$ & $6(5-7)$ & $6(5-7)$ & $6(4-7)$ \\
\hline Increasing the bend in your surgical knee & $4(2-6)$ & $7(6-7)$ & $6(5-7)$ & $6(5-7)$ \\
\hline Increasing the straightening in your surgical knee & $4(I-5)$ & $6(5-7)$ & $5(4-6)$ & $6(3-6)$ \\
\hline Increasing the strength in your legs & $6(4-7)$ & $6(5-7)$ & $6(5-7)$ & $6(5-6)$ \\
\hline
\end{tabular}

\footnotetext{
* International Classification of Functioning, Disability, and Health

t median $\left(25^{\text {th }}\right.$ to $75^{\text {th }}$ percentile); importance ratings are on a 7 -point scale $(I=$ not important, $7=$ important to a very great extent $)$
} 
Table 3: Importance levels for patient concerns linked to the ICF* Activity component: pre-operatively (pre-op) and two, four and six weeks after knee arthroplasty $(n=54)$

\begin{tabular}{|c|c|c|c|c|}
\hline Patient Concern & Pre-op & Week 2 & Week 4 & Week 6 \\
\hline Getting out of bed on your own & $4.5(3-7)$ & $6(5-7)$ & $5(3-6)$ & $5(3-7)$ \\
\hline Getting in/out of bath & $5(3-7)$ & $I(I-4)$ & $5(3-6)$ & $4(2-6)$ \\
\hline Putting on your own shoes or socks & $5(3-6)$ & $6(4-7)$ & $5(4-6)$ & $5(4-6)$ \\
\hline Dressing yourself & $4(2-6)$ & $5.5(4-7)$ & $5.5(4-6)$ & $6(4-7)$ \\
\hline Walking on a flat surface & $5(4-7)$ & $6(5-7)$ & $6(4-7)$ & $6(4-7)$ \\
\hline Walking on uneven ground & $5(3-7)$ & $1(1-3)$ & $3(I-5)$ & $4(3-6)$ \\
\hline Descending stairs & $6(4-7)$ & $3.5(I-6)$ & $5(3-6)$ & $5(4-6)$ \\
\hline Ascending stairs & $6(5-7)$ & $4(I-6)$ & $5(3-6)$ & $5(4-6)$ \\
\hline Cooking your own meals & $5(2-7)$ & $I(I-3)$ & $4(2-6)$ & $4(2-6)$ \\
\hline Doing your own housework & $5(3-6)$ & $I(I-3)$ & $3(2-6)$ & $4(2-6)$ \\
\hline Heavy domestic duties & $2.5(1-6)$ & $I(1-1)$ & $I(I-2)$ & I (I-3) \\
\hline Light domestic duties & $4(3-6)$ & $2(I-3)$ & $4(3-5)$ & $4.5(3-6)$ \\
\hline Getting in/out of car & $6(4-7)$ & $4(2-6)$ & $6(4-7)$ & $6(5-7)$ \\
\hline Comfortably sit in car & $5(3-6)$ & $4(2-6)$ & $6(4-6)$ & $5.5(4-6)$ \\
\hline Doing your exercises as prescribed by your PT & $4(I-7)$ & $7(6-7)$ & $6(6-7)$ & $6(5-7)$ \\
\hline
\end{tabular}

* International Classification of Functioning, Disability, and Health

† median ( $25^{\text {th }}$ to $75^{\text {th }}$ percentile); importance ratings are on a 7-point scale $(I=$ not important, $7=$ important to a very great extent)

over time while others do not. In the discussion that follows we suggest these changes reflect the clinical aspects of the delivery of care and the early recovery process. We also propose that the importance ratings provide direct evidence that supports the differentiation of the Activity and Participation components of the ICF in the clinical context of this study. To the best of our knowledge, this is the first study of this nature.

The Friedman's ANOVA revealed the importance rankings of all four ICF components changed over time. Post hoc testing showed that concerns in the ICF Body Function component were ranked higher at post-operative week two compared to before surgery. The WHO has defined Body Function [10] as physiological functions of the body systems including psychological functions. Following major joint surgery such as TKA, physiological functions perceived as important by patients, such as motion of the knee joint, strength in the lower extremities and sleeping at night are commonly impaired for the first month postoperatively [6]. The sensation of pain and swelling in the surgical leg are also common occurrences during this phase of post-operative recovery. Presence of these impairments after surgery would naturally cause discomfort and limit normal daily activities. Consistent with this logic, patients in this study rated these Body Function concerns as being more important to them in the first month following surgery.

Going through surgery and recovery may also explain the pattern of change in the Activity and Participation importance ratings. Post hoc testing showed that patients ranked concerns in these components as being less important two weeks after surgery. Due to the nature of the surgery it is

Table 4: Importance levels for patient concerns linked to ICF* Participation and Environmental Factors components: pre-operatively (pre-op) and two, four and six weeks after knee arthroplasty $(n=54)$

\begin{tabular}{|c|c|c|c|c|}
\hline Patient Concern by ICF component & Pre-op & Week 2 & Week 4 & Week 6 \\
\hline \multicolumn{5}{|l|}{ Participation } \\
\hline Driving a vehicle & $6(3-7)$ & $I(I-1)$ & I (I-4) & $3.5(1-6)$ \\
\hline Going shopping & $5(3-6)$ & $I(I-I)$ & $3(I-5)$ & $4(3-6)$ \\
\hline Returning to your hobbies & $5(2-7)$ & $I(I-2)$ & $2(1-3)$ & $3(1-5)$ \\
\hline Going back to your regular exercise class or sport & $4(1-7)$ & $I(I-I)$ & $I(1-4)$ & $3(1-6)$ \\
\hline \multicolumn{5}{|l|}{ Environmental factors } \\
\hline Being less of a burden on your spouse or caregiver & $6(4-7)$ & $6(5-7)$ & $6(5-7)$ & $6(5-7)$ \\
\hline Having the support of your family members & $6(5-7)$ & $6(6-7)$ & $6(5-7)$ & $6(5-7)$ \\
\hline Having the support of your neighbours & $3(1-5)$ & $3(\mathrm{I}-5)$ & $4(1-5)$ & $4(1-6)$ \\
\hline Receiving competent care from health care workers in a timely manner & $2.5(1-6)$ & $7(6-7)$ & $6(5-7)$ & $6(4-7)$ \\
\hline
\end{tabular}

* International Classification of Functioning, Disability, and Health

t median $\left(25^{\text {th }}\right.$ to $75^{\text {th }}$ percentile); importance ratings are on a 7 -point scale $(I=$ not important, $7=$ important to a very great extent) 


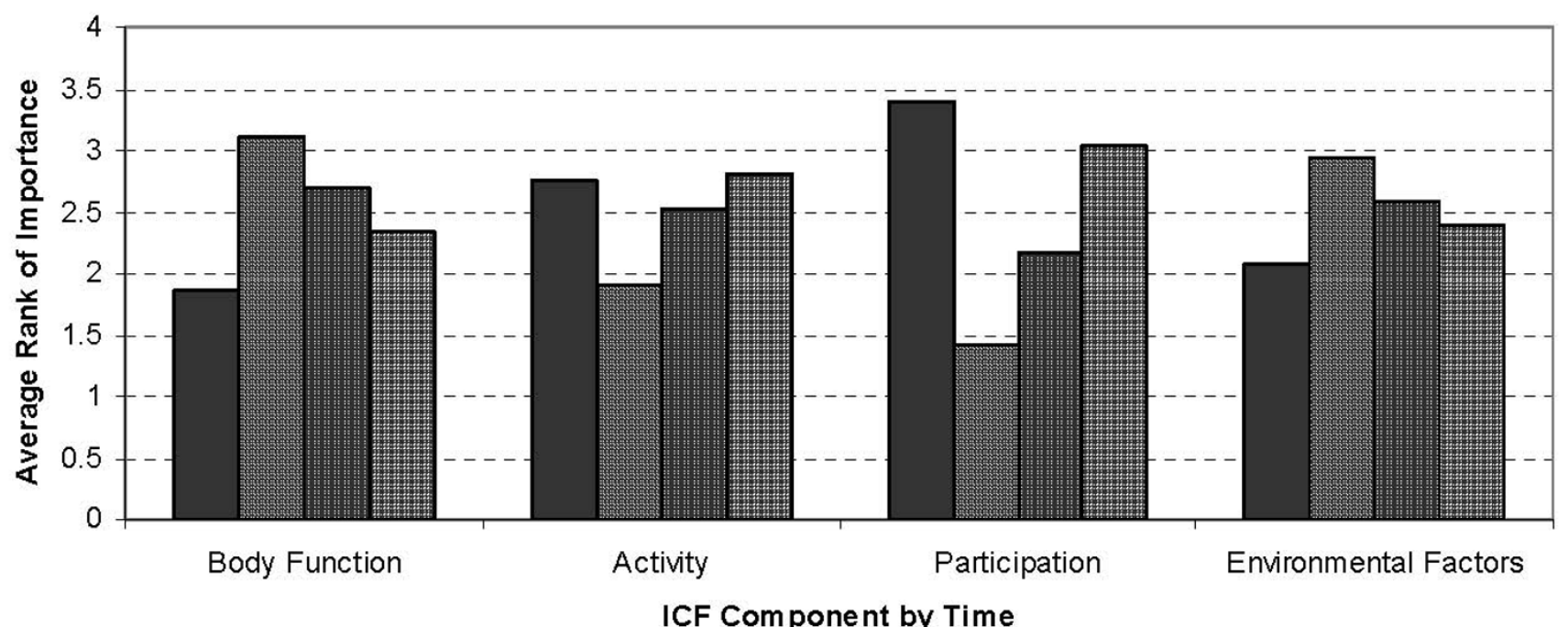

Pre-op 2 wks post-op $\mathbf{0} 4$ wks post-op $\mathbf{0} 6$ wks post-op

Figure 2

Average rank of importance for patient concerns grouped by ICF* component: pre-operatively (pre-op) and two, four and six weeks after (post-op) primary total knee arthroplasty $(\mathbf{n}=\mathbf{5 4})$. * International Classification of Functioning, Disability, and Health

common for patients to experience impairments with certain body functions (increased pain and swelling, decreased knee range of motion, impaired sleep), which generally lead to limitations in the activities that they can perform. Researchers have reported a decline in physical function in the first month following TKA surgery [6]. Consistent with this, patients in our study demonstrated significant decreases in the KOOS ADL and Sport/Rec subscales in the first two weeks after surgery. Due to this decline, concerns from the ICF Activity component (e.g. 'walking on uneven ground', 'cooking your own meals', 'doing your own housework') and concerns from the Participation component (e.g. 'driving a vehicle', 'shopping' or 'returning to hobbies') dropped in importance during the first two weeks post-operatively. Their subsequent increase in importance to pre-operative levels after surgery, may reflect the fact that patients are generally starting to feel better, are mobilizing with greater ease and are becoming less dependent on their caregivers. Indeed, Kennedy and colleagues [22] and Stratford and Kennedy [23] found that physical function improved to pre-operative levels by post-operative week six to eight. Again, consistent with this, patients in our study showed significant increases in the KOOS ADL and Sport/Rec subscales from two weeks through to six weeks post-operatively. The importance ratings for Body Function, Activity and Participation, combined with parallel changes in these KOOS subscale scores therefore, supports the validity of these concerns and their importance to patients because they mirror the surgical and early rehabilitation focus.

Just like the Body Function component, patient concerns that mapped to the Environmental Factors component were ranked higher two weeks after surgery. Due to the immediate decline in physical function [6], patients require the support of family members and health care workers to help them manage with daily activities and restore their physical function. Consistent with this, concerns such as 'having the support of your family members', 'receiving competent care from health care workers' and 'being less of a burden on your spouse or caregiver' were important to patients during this acute post-operative phase of their recovery. 'Having the support of your neighbours' was the only concern mapping to the Environmental Factors component that was of moderate to small importance at each post-operative evaluation session. This may relate to the fact that TKA is an elective surgery, which allows the patient time to plan for their postoperative needs using alternative resources (home care, family members etc.) and therefore, not requiring the support of their neighbours.

Two concerns that were not covered by the ICF components, 'receiving appropriate information regarding what to expect with rehabilitation following your surgery' and 'being independent' were of great importance to patients 
before surgery and remained at the same level of importance throughout the first six weeks of recovery. The first emphasizes the importance of education from the patient perspective. In this regard, Soever and MacKay [24] have documented that receiving information about their rehabilitation is important to patients and that this type of education improves patient satisfaction following total joint replacement surgery. The second highlights the consistently high value that patients place on independence. Hinojosa and Youngstrom have reported that "independence is defined by the individual's culture and values, support systems, and ability to direct his or her life" [25]. Gignac and Cott [26] have reported that a loss of independence may have consequences on the quality of life and psychosocial well being of an individual. The cultural background of patients in this study and the support system in our society along with the consequences of losing independence, may explain why patients consistently rated the importance of this concern so high, throughout the first six weeks of recovery.

There is considerable debate regarding the need to distinguish between the Activity component and the Participation component of the ICF [12-14,27]. While the WHO decided not to distinguish between these two components; others have stressed the importance of their differentiation if the ICF is to be widely used when describing health and health-related states of various populations across the world [12-14]. The results of this study highlight the challenge one faces in differentiating the ICF Activity and Participation components. On one hand, the average ranking of importance across time for these two components (Figure 2) reveals a similar pattern over time, suggesting there is little to be gained from separation of the ICF components when describing the importance of patient concerns about recovery. On the other hand, the relatively low values in the KOOS Sport/Rec subscale (Figure 1) may explain the pattern in Participation concerns after the two-week mark following surgery. Only those concerns in the ICF Participation component showed consistent increases in their level of importance through the first six weeks after surgery. We believe this highlights one tangible benefit from separating the two components in the context of this work: patients are starting to think about their return to participation in roles early in the recovery phase. As evidenced by the KOOS Sport/Rec subscale, they are also aware of their limitations in Participation-related activities. This is important for the clinician to note as researchers have shown that patient expectations are a predictor of functional outcome and satisfaction following total joint replacement surgery [28]. Therefore, patient education should encompass return to participation roles very soon after surgery and in particular, should re-evaluate participation-related goals from two to six weeks of postoperative care. Future studies should deter- mine if the increasing importance assigned to Participation concerns in this study continues to increase beyond the six-week mark post-operatively.

All participants in the study were recruited from a single tertiary care hospital. This may be viewed as decreasing the generalizability of this study to other settings. Even though the results may not be applicable to all TKA populations, the patient demographics and pre-operative functional status findings were comparable to that of other TKA studies [17,29-31]. The rehabilitation setting (e.g. home care, in-patient or out-patient facilities) following surgery may influence what is important to patients. The majority of patients in this study received their initial therapy at home after acute care discharge until approximately three weeks following surgery. Then they continued therapy at an out-patient clinic of their choice. Therefore, it is possible that patients undergoing TKA, who receive therapy in a different setting than described above may have different priorities and concerns during the first six weeks following surgery. We note however that no subjects in this study provided additional concerns when given the opportunity to do so at four different time points of data collection. Furthermore, when the importance findings are viewed in combination with the KOOS data, changes in patient concerns mirrored the early recovery pattern from TKA surgery. This supports the validity of the concerns we investigated and combined with the finding that importance levels varied over time, suggests a temporal element that should be included in future work of this nature. As there was no test-retest component in this study, reliability of the importance ratings at each time point of data collection could not be confirmed. Another limitation of this study was the inability to include non-English speaking individuals. Therefore, the results are not applicable to non-English speaking recipients of TKA surgery. Finally, our sample size for this study was based on sample size calculations of a concurrent study of responsiveness of the WOMAC [unpublished]. This seemed acceptable because we had no previous importance ratings on which to base our sample size calculations. Where no significant differences were found, it may be possible the study was underpowered.

\section{Conclusion}

In conclusion the study shows the level of importance that patients assign to concerns about recovery from TKA surgery, is a reflection of the stage they are in, during the surgical and rehabilitation process. In particular, Participation restrictions become increasingly important to patients from two to six weeks after surgery, suggesting that patients do think about their return to activities at the societal level early in their postoperative rehabilitation. Clinicians can use this knowledge to integrate their approach to education, goal setting and managing expec- 
tations about return to participation roles after TKA surgery.

\section{Competing interests}

The authors declare that they have no competing interests.

\section{Authors' contributions}

$\mathrm{RR}, \mathrm{AMD}$ and BMC designed the study. RR collected and analyzed the data and drafted the manuscript with regular feedback from AMD and BMC. All authors read and approved the final manuscript.

\section{Acknowledgements}

This work was supported by a Premier's Research Excellence Award from the Ontario Ministry of Health and Long-term Care to Dr. Davis and by the Dr. Jal Tata Research Award from the London district of the Ontario Physiotherapy Association to Ravi Rastogi.

The study was completed by Ravi Rastogi in partial fulfilment of the requirements for the degree of Master of Science at the School of Physical Therapy, The University of Western Ontario.

\section{References}

I. Davis MA, Ettinger WH, Neuhaus JM, Mallon KP: Knee osteoarthritis and physical functioning: Evidence from the NHANES I epidemiologic followup study. J Rheumatol I99|, I8:59|-598.

2. Hartley RC, Barton-Hanson NG, Finley R, Parkinson W: Early patient outcomes after primary and revision total knee arthroplasty. J Bone Joint Surg [Br] 2002, 84:994-999.

3. Heck DA, Robinson RL, Partridge CM, Lubitz R, Freund DA: Patient outcomes after knee replacement. Clin Orthop Relat Res 1998, I:93-1 I0.

4. Canadian Institute for Health Information (ClHI): Canadian Joint Replacement Registry (CJRR). Hip and Knee Replacements in Canada 2006. Report.

5. Dickstein R, Heffes Y, Shabtai E, Markowitz E: Total knee arthroplasty in the elderly: Patients' self-appraisal 6 and 12 months postoperatively. Gerontology 1998, 44:204-210.

6. Salmon P, Hall GM, Peerbhoy D, Shenkin A, Parker C: Recovery from hip and knee arthroplasty: Patients' perspective on pain, function, quality of life and well-being up to 6 months postoperatively. Arch Phys Med and Rehabil 200I, 82:360-366.

7. Noble PC, Conditt MA, Cook KF, Mathis KB: The John Insall Award: Patient expectations affect satisfaction with total knee arthroplasty. Clin Orthop Relat Res 2006, 452:35-43.

8. McFarlane A, Brooks P: The assessment of disability and handicap in musculoskeletal disease. I Rheumatol 1997, 24(5):985-989.

9. Newman S: Psychosocial measures in musculoskeletal trials. J Rheumatol 1997, 24(5):979-984.

10. World Health Organization: International Classification of Functioning, Disability and Health. 200I.

II. Stucki G, Cieza A, Ewert T, Kostanjsek N, Chatterij S, Ustun TB: Application of the International Classification of Functioning, Disability and Health (ICF) in clinical practice. Disabil Rehabil 2002, 24:28I-282.

12. Whiteneck G: Conceptual models of disability: past, present, and future. Workshop on Disability in America: A new look - summary and background papers. Edited by: Field MJ, Jette AM, Martin L. The National Academic Press, Washington DC; 2006. Appendix B:50-66.

13. Jette AM, Haley SM, Kooyoomjian JT: Are the ICF activity and participation dimensions distinct? I Rehabil Med 2003, 35:145-149.

14. Perenboom RJM, Chorus AMJ: Measuring participation according to the International Classification of Functioning, Disability and Health (ICF). Disabil Rehabil 2003, 25:577-587.
15. Rastogi R, Davis AM, Chesworth BM: Patient concerns in the first six weeks following primary total knee arthroplasty. Health Qual Life Outcomes 2007, 5:48. doi: 10.1 186/1477-7525-5-48.

16. Farrar JT, Young JP Jr, LaMoreaux L, Werth JL, Poole RM: Clinical importance of changes in chronic pain intensity measured on an II-point numerical pain rating scale. Pain 200I, 94:I49-I58.

17. Roos EM, Toksvig-Larsen S: Knee injury and osteoarthritis outcome score (KOOS) - validation and comparison to the WOMAC in total knee replacement. Health Qual Life Outcomes 2003, I: 17-26.

18. Ethgen O, Bruyere O, Richy F, Dardennes C, Reginster J-V: Healthrelated quality of life in total hip and total knee arthroplasty. A qualitative and systematic review of the literature. J Bone Joint Surg $[\mathrm{Am}]$ 2004, 86(5):963-74.

19. Sangha O, Stucki G, Liang MH, Fossel AH, Katz JN: The self-administered comorbidity questionnaire: $A$ new method to assess comorbidity for clinical and health services research. Arthritis Care Res 2003, 49:156-163.

20. Portney LG, Watkins MP: Foundations of Clinical Research: Applications to Practice. 2nd edition. New Jersey; Prentice Hall Health; 2000.

21. Winer BJ: Single-factor experiments having repeated measures on the same elements. In Statistical principles in experimental design 2nd edition. New York: McGraw-Hill Book Company; 1971:261-308.

22. Kennedy DM, Stratford PW, Wessel J, Gollish JD, Penney D: Assessing stability and change of four performance measures: a longitudinal study evaluating outcome following total hip and knee arthroplasty. BMC Musculoskelet Disord 2005, 6:3-I4.

23. Stratford PW, Kennedy DM: Performance measures were necessary to obtain a complete picture of osteoarthritic patients. J Clin Epidemiol 2006, 59:160-167.

24. Soever L, Mackay C: Best practices across the continuum of care for total joint replacement. Greater Toronto Area Rehabilitation Network. Final Report 2005.

25. Hinojosa J, Youngstrom MJ: Position paper: broadening the construct of independence. Am J Occup Ther 2002, 56(6):660.

26. Gignac MA, Cott C: A conceptual model of independence and dependence for adults with chronic physical illness and disability. Soc Sci Med 1998, 47(6):739-753.

27. Dahl TH: An introduction and discussion of its potential impact on rehabilitation services and research. J Rehabil Med 2002, 34:20I-204.

28. Mahomed NN, Liang MH, Cook EF, Daltroy LH, Fortin PR, Fossel AH, Katz JN: The importance of patient expectations in predicting functional outcomes after total joint arthroplasty. I Rheumatol 2002, 29(6): $1273-1279$.

29. Stratford PW, Kennedy DM, Hanna SE: Condition-specific Western Ontario McMaster Osteoarthritis Index was not superior to region-specific Lower Extremity Functional Scale at detecting change. J Clin Epidemiol 2004, 57:1025-1032.

30. Bachmeier CJM, March LM, Cross MJ, Lapsley HM, Tribe KL, Courtenay BG, Brooks PM: A comparison of outcomes in osteoarthritis patients undergoing total hip and knee replacement surgery. Osteoarthritis Cartilage 200I, 9: I37-I 46.

3I. Lingard EA, Katz JN, Wright RJ, Wright EA, Sledge CB, Kinemax Outcomes group: Validity and responsiveness of the Knee Society Clinical Rating System in comparison with the SF-36 and WOMAC. J Bone Joint Surg [Am] 200I, 83:1856-1864. 